\title{
Immunoprotective Steroids and SHBG in Non-Treated Hypothyroidism and their Relationship to Autoimmune Thyroid Disorders
}

\author{
K. DRBALOVÁ, P. MATUCHA, M. MATĚJKOVÁ-BĚHANOVÁ, R. BÍLEK, L. KŘíŽ, \\ H. KAZIHNITKOVÁ, R. HAMPL
}

Institute of Endocrinology, Prague, Czech Republic

Received November 12, 2007

Accepted January 28, 2008

On-line February 13, 2008

\begin{abstract}
Summary
Immunomodulatory steroids, dehydroepiandrosterone and its 7hydroxylated metabolites and sex hormone-binding globulin (SHBG) were determined in sera of 88 women aged $18-75$ years. The group consisted of 34 healthy women, 37 women with subclinical and 17 women with manifest hypothyroidism. In all subjects the laboratory parameters of thyroid function (thyrotropin, free thyroxine and triiodothyronine) and thyroid autoantibodies to thyroid peroxidase and thyroglobulin were determined. The aim was to find out 1 ) whether the above steroids and SHBG levels differ in individual groups according to thyroid status, 2) whether correlations exist among investigated steroids and thyroid laboratory parameters, and 3) whether the respective steroid and SHBG levels differ according to the presence of principal thyroid autoantibodies. With the exception of $7 \beta$-hydroxy-dehydroepindrosterone levels, which were decreased in patients with manifest hypothyroidism $(p<0.05)$, no significant differences in steroid and SHBG levels among groups according to diagnosis were found. On the other hand, significantly decreased levels of all the immunomodulatory steroids studied were found in subjects with positive titres of thyroid autoantibodies. This finding was supported by a tight negative correlation among the above steroids and thyroid autoantibodies. In addition, these steroids correlated negatively with thyrotropin and positively with free thyroid hormones. The results point to a negative relationship between the above mentioned immunoprotective steroids and the extent of the autoimmune process in hypothyroidism.
\end{abstract}

\section{Key words}

Hypothyroidism - Autoimmunity - Dehydroepiandrosterone • 7-hydroxy-dehydroepiandrosterone • SHBG

\section{Corresponding author}

R. Hampl, Institute of Endocrinology, Národní třída 8, 11694 Praha 1, Czech Republic. E-mail: rhampl@endo.cz

\section{Introduction}

Dehydroepiandrosterone (DHEA) and its 7hydroxylated metabolites (7-OH-DHEA) are believed to act as locally active immunoprotective agents, attenuating some immunosuppressive effects of glucocorticoids (Šulcová et al. 2001, Morfin 2002, Muller et al. 2006). The involvement of sex hormone-binding globulin (SHBG) in the thyroid axis and its positive correlation with thyroid hormone levels due to stimulation of its synthesis in liver by thyroid hormones is well known and it was even suggested as an additional marker for monitoring the treatment of thyroid disorders (Brenta et al. 1999). Hypothyroidism, including its subclinical form, is an important issue today in regards to its early diagnostics and monitoring of adequate treatment (Vanderpump and Tunbridge 2002, Wilson and Curry 2005). In the search for additional laboratory markers which would enable us to refine the diagnostics of hypothyroidism, we have focused on the above mentioned immunomodulatory steroids and SHBG in relation to thyroid laboratory parameters reflecting thyroid gland function and the extent of the autoimmune process. The following aims were addressed: 1) to find out whether the above mentioned steroids and SHBG levels differ in individual groups according to thyroid status (manifest and subclinical hypothyroidism and healthy controls), 2) whether correlations exist among the investigated steroids and thyroid laboratory parameters, and 3) whether the respective steroid and SHBG levels differ according to the presence of thyroid autoantibodies. 


\section{Materials and Methods}

\section{Subjects}

The studied group consisted of 88 women from a broad age range (18-75 years). Out of them, 34 were healthy euthyroid women (18-70 years), 38 patients had subclinical hypothyroidism aged 18-83 years, diagnosed on the base of serum levels of thyrotropin (TSH) and free thyroid hormones (fT4, fT3), without overt endocrine disorders, and 17 women, aged 27-75 years, showed manifest hypothyroidism confirmed by ultrasonography. All subjects had not received prior treatment for thyroid or other endocrine disorders and were coming to the Institute of Endocrinology for the first time when participating in our study.

Blood samples were drawn from the cubital vein between 7-8 h a.m., obtained sera were analyzed for the hormones and other analytes listed below. All analyses were a part of routine laboratory testing for thyroid disorders.

\section{Laboratory tests}

Thyroid parameters, serum thyroid stimulating hormone, thyrotropin, (TSH, normal range 0.27-4.30 $\mathrm{mIU} / \mathrm{l}$ ), free thyroxine ( $\mathrm{fT}_{4}$, normal range 12.0-22.0 $\mathrm{pmol} / \mathrm{l}$ ) and free trioiodothyronine ( $\mathrm{fT}_{3}$, normal range 2.80-7.10 pmol/1) were measured by ECLIA from Roche Diagnostics GmBH, Mannheim, Germany, using commercial Elecsys System 2010. The intra-assay- and inter-assay variability were 3.0, 2.0 and 2.0, and 7.2, 4.8 and 3.4 for $\mathrm{TSH}_{1} \mathrm{fT}_{4}$, and $\mathrm{fT}_{3}$, respectively. Anti thyroid peroxidase (AntiTPO) and anti thyroglobulin autoantibodies (AntiTg), physiological levels below 25 and $125 \mathrm{U} / \mathrm{ml}$, respectively, were assessed by enzymelinked immunosorbent assay (ELISA) (Aesku. Diagnostics, Wendelsheim, FRG). The intra-assay- and inter-assay coefficients of variation were 5.4 and 5.6, and 8.4, and 8.6, for AntiTPO and AntiTg, respectively. Dehydroepiandrosterone, its sulfate, and SHBG were measured by commercial radioimmunoassay and immunoradiometric kits from Immunotech (Czech Division, Marseille, France). Physiological levels of the latter analytes strongly depended on age and our data agreed with those reported by the manufacturer. $7 \beta$ Hydroxydehydroepiandrosterone (7 $\beta-\mathrm{OH}-\mathrm{DHEA})$ and its $7 \alpha$-hydroxyisomer ( $7 \alpha-\mathrm{OH}$-DHEA) were determined by the in-the-laboratory developed radioimmunoassays (Lapčík et al. 1999, Lapčík et al. 1998). The intra-assay-, and inter-assay variability, for DHEA, DHEAS, $7 \alpha-\mathrm{OH}-$ DHEA, 7ß-OH-DHEA, and SHBG were 7.2, 4.2, 7.1, $6.7,6.1 \%$, and $11.0,7.2,10.6,10.0$, and $7.9 \%$, respectively.

\section{Statistics}

The differences among the groups were evaluated by analysis of covariance (ANCOVA) with age as a covariate, using the statistical software Statgraphics Plus version 7 (Manugistics Inc., Rockville, MA, USA). Spearman and Pearson correlation analyses were applied for evaluation of mutual relationships between laboratory data. $P$ value less than 0.05 was considered to indicate significance.

\section{Results}

Table 1 shows the basic statistical data concerning thyroid hormone levels, two major thyroid autoantibodies, DHEA, its sulfate, both 7-hydroxylated DHEA metabolites and SHBG in three groups of women - healthy controls and untreated patients with subclinicaland manifest hypothyroidism. The significant differences from the control group at the 95 and $99 \%$ levels are marked with asterisks. Besides expected differences in thyroid hormones and thyroid autoantibodies, the only significant difference $(P<0.05)$ in steroid levels was decreased $7 \beta-O H-D H E A$ in women with manifest hypothyroidism. The decreased levels of SHBG in both hypothyroid groups were insignificant.

As demonstrated in Table 2, when all 88 subjects were divided into subgroups according to positivite and negativite autoantibody titres (AntiTPO and AntiTg negative, AntiTPO positive, both AntiTPO and AntiTg positive), three immunomodulatory steroids (DHEA, $7 \alpha-$ and $7 \beta-O H-D H E A)$ showed significantly decreased levels when compared with controls. In healthy women, only 5 out of $34(14.7 \%)$ had positive titre of at least one autoantibody, while in patients with subclinical hypothyroidism there were 24 women $(64.9 \%)$, and in women with manifest hypothyroidism only out of seventeen one had negative titres of both autoantibodies. No differences among these subgroups were found in SHBG levels. 


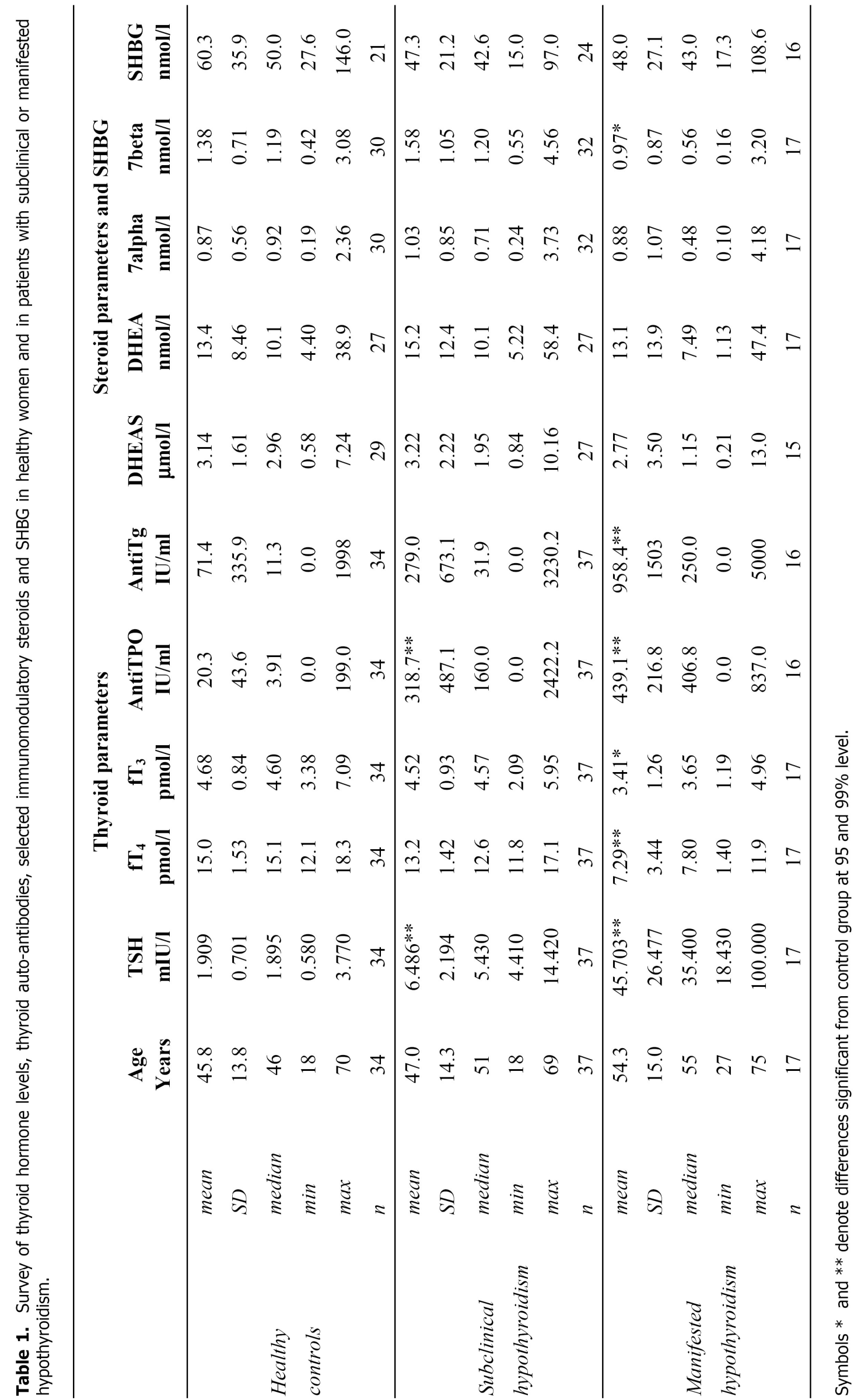




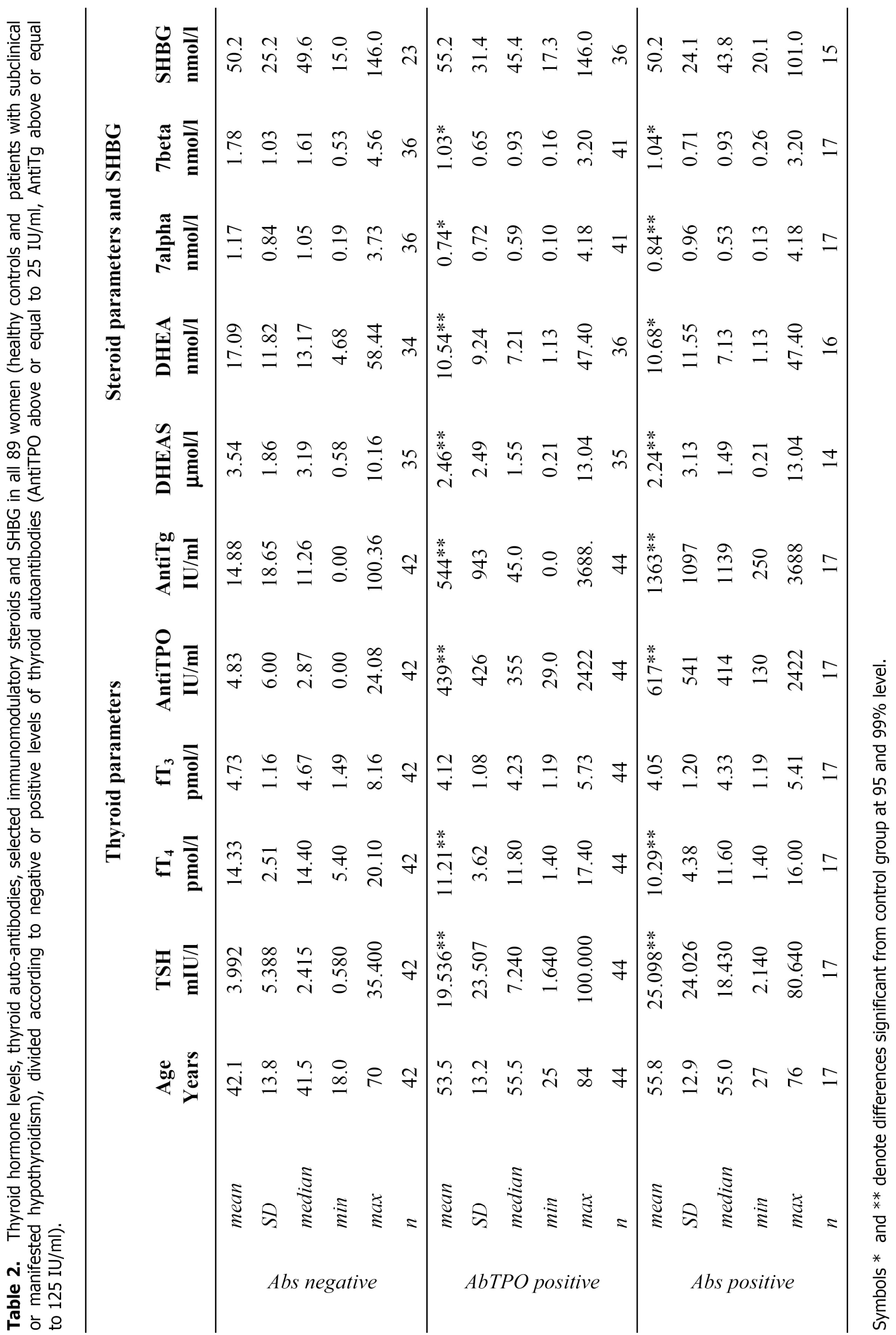


Table 3. Correlation matrix of thyroid hormone parameters, thyroid autoantibodies and selected immunomodulatory steroid in all 88 women (healthy controls and patients with subclinical or manifested hypothyroidism). Above the diagonal (upper right part): Pearson's correlations, below the diagonal (lower left part): Spearman's correlations. Each cell from above represents the correlation coefficient $(r)$, number of correlated pairs $(n)$ and significance $(P$ value $)$ and. Significant correlations are in bold.

\begin{tabular}{|c|c|c|c|c|c|c|c|c|c|}
\hline & TSH & $\mathrm{fT}_{4}$ & $\mathrm{fT}_{3}$ & AntiTPO & AntiTg & DHEAS & DHEA & 7alpha & 7 beta \\
\hline \multirow{3}{*}{ TSH } & & -0.838 & -0.338 & 0.740 & 0.269 & -0.323 & -0.389 & -0.082 & -0.358 \\
\hline & & 31 & 31 & 31 & 31 & 31 & 31 & 31 & 31 \\
\hline & & 0.000 & 0.063 & 0.000 & 0.143 & 0.077 & 0.031 & 0.662 & 0.048 \\
\hline \multirow{3}{*}{$f T 4$} & -0.838 & & 0.454 & -0.565 & -0.025 & 0.230 & 0.321 & -0.034 & 0.228 \\
\hline & 46 & & 31 & 31 & 31 & 31 & 31 & 31 & 31 \\
\hline & 0.000 & & 0.010 & 0.001 & 0.893 & 0.213 & 0.078 & 0.858 & 0.218 \\
\hline \multirow{3}{*}{$f T_{3}$} & -0.364 & 0.498 & & -0.414 & -0.044 & 0.364 & 0.356 & 0.164 & 0.421 \\
\hline & 46 & 46 & & 31 & 31 & 31 & 31 & 31 & 31 \\
\hline & 0.015 & 0.001 & & 0.021 & 0.813 & 0.044 & 0.050 & 0.378 & 0.018 \\
\hline \multirow{3}{*}{ AntiTPO } & 0.722 & -0.630 & -0.351 & & 0.515 & -0.339 & -0.407 & -0.253 & -0.436 \\
\hline & 46 & 46 & 46 & & 31 & 31 & 31 & 31 & 31 \\
\hline & 0.000 & 0.000 & 0.019 & & 0.003 & 0.063 & 0.023 & 0.171 & 0.014 \\
\hline \multirow{3}{*}{ AntiTg } & 0.336 & -0.205 & -0.112 & 0.606 & & -0.009 & -0.008 & -0.094 & -0.030 \\
\hline & 46 & 46 & 46 & 46 & & 31 & 31 & 31 & 31 \\
\hline & 0.024 & 0.170 & 0.452 & 0.000 & & 0.964 & 0.967 & 0.614 & 0.875 \\
\hline \multirow{3}{*}{ DHEAS } & -0.396 & 0.414 & 0.382 & -0.369 & -0.065 & & 0.876 & 0.469 & 0.688 \\
\hline & 46 & 46 & 46 & 46 & 46 & & 31 & 31 & 31 \\
\hline & 0.008 & 0.006 & 0.011 & 0.013 & 0.662 & & 0.000 & 0.008 & 0.000 \\
\hline \multirow{4}{*}{ DHEA } & -0.418 & 0.440 & 0.341 & -0.435 & -0.123 & 0.888 & & 0.440 & 0.690 \\
\hline & 46 & 46 & 46 & 46 & 46 & 46 & & 31 & 31 \\
\hline & 0.005 & 0.003 & 0.022 & 0.004 & 0.411 & 0.000 & & 0.013 & 0.000 \\
\hline & -0.249 & 0.278 & 0.240 & -0.390 & -0.168 & 0.651 & 0.670 & & 0.841 \\
\hline \multirow[t]{3}{*}{ 7alpha } & 46 & 46 & 46 & 46 & 46 & 46 & 46 & & 31 \\
\hline & 0.095 & 0.062 & 0.108 & 0.009 & 0.260 & 0.000 & 0.000 & & 0.000 \\
\hline & -0.427 & 0.449 & 0.385 & -0.511 & -0.108 & 0.784 & 0.781 & 0.876 & \\
\hline \multirow[t]{2}{*}{7 beta } & 46 & 46 & 46 & 46 & 46 & 46 & 46 & 46 & \\
\hline & 0.004 & 0.003 & 0.010 & 0.001 & 0.470 & 0.000 & 0.000 & 0.000 & \\
\hline
\end{tabular}

Finally, the data from all women were mutually correlated. Table 3 shows the correlation matrix for thyroid hormone parameters, thyroid autoantibodies and immunomodulatory steroids in all 88 women. Besides the expected correlations among thyroid parameters on one side, and DHEA, its sulfate and its 7-hydroxylated metabolites on the other, the following significant correlations were found between thyroid parameters and investigated steroids: DHEAS, DHEA and 7 $\beta-\mathrm{OH}-\mathrm{DHEA}$ negatively correlated with $\mathrm{TSH}$, in the case of the latter two steroids when using both statistical approaches. On the other hand all of these steroids correlated positively with free thyroid hormones, as $\mathrm{fT}_{3}$ concerns, using both statistical methods. DHEA, 7 $\beta-O H-D H E A$, DHEAS and also $7 \alpha-\mathrm{OH}-\mathrm{DHEA}$ levels negatively correlated with autoantibodies to thyroid peroxidase (AntiTPO), the latter two steroids only when using Sperman's analysis.

\section{Discussion}

With respect to the autoimmune origin of most of the thyroid disorders, this study is the first attempt to establish a relationship between thyroid laboratory parameters, including major thyroid autoantibodies and DHEA and its 7-hydroxylated metabolites, believed to act as immunoprotective agents (Šulcová et al. 2001, Morfin 2002, Muller et al. 2006). Recently we have demonstrated that administration of 7-oxo-DHEA, one of the candidates of steroid replacement therapy to healthy male volunteers influenced temporarily actual levels of thyroid hormones (Hampl et al. 2006). In addition, SHBG levels were investigated due to its involvement in 
thyroid axis (Brenta et al. 1999). The studied groups consisted either of healthy women or women with subclinical- or manifest hypothyroidism, confirmed clinically.

Comparison of the above steroid levels in both patient's groups and healthy subjects revealed only decreased levels of one of the 7-hydroxylated DHEA metabolites, 7 $\beta$-OH-DHEA in patients with manifest hypothyroidism. We found decreased levels of 7hydroxylated metabolites in our preliminary study of patients with Hashimoto thyreoiditis (Hampl et al. 1999) and our new data confirms this early finding. Surprisingly, the decreased level of SHBG in hypothyroid groups was insignificant. This finding, however, is consistent with our earlier report addressing the diagnostic value of SHBG determination in patients with various thyroid disorders. The serum levels of this protein were significantly increased in patients with hyperthyroidism, including its subclinical form, but the decrease of this protein's levels in hypothyroid subjects was insignificant (Hampl et al. 2003)

On the other hand, all of the immunoprotective steroid levels were significantly lower in subjects with positive titres of major thyroid autoantibodies, the occurrence of which was markedly increased in hypothyroidism. This finding was further supported by the tight negative correlation of DHEA and its 7- hydroxylated metabolites with AbTPO levels. This is also in agreement with a negative correlation of these steroids with TSH, along with their positive correlation with both free thyroid hormones.

The negative association of autoimmune thyroid disorders with immunoprotective DHEA metabolites has not yet been reported. In connection with this, it is of interest to note a recent report which showed a protective effect of DHEA and its $7 \alpha$-hydroxylated metabolite against another autoimmune disorder, an experimentally induced colitis, despite the fact that the mechanism of the latter effect may be different from that which is probably operating in thyroid autoimmune disorders (Pélissier et al. 2006).

In conclusion, our data clearly demonstrates, for the first time, a negative relationship between the levels of the above mentioned immunoprotective steroids and the extent of the autoimmune process, at least in hypothyroidism.

\section{Conflict of Interest}

There is no conflict of interest.

\section{Acknowledgements}

The work was supported by the Grant No. 9160-3 from the Internal Grant Agency of the Czech Ministry of Health.

\section{References}

BRENTA G, SCHNITMAN M, GURFINKIEL M, DAMILANO S, PIERINI A, SINAY I PISAREV MA: Variations of sex hormone-binding globulin in thyroid dysfunctions. Thyroid 9: 273-277, 1999.

HAMPL R, LAPČÍK O, HILL M, STÁRKA L: 7-Hydroxylated metabolites of dehydroepiandrosterone as possible neuroactive and immunomodulatory steroids and development of their radioimmunoassy. J Physiol Lond 518: 14P-15P, 1999.

HAMPL R, KANCHEVA R, HILL M, BIČÍKOVÁ M, VONDRA K: Interpretation of sex hormone-binding globulin levels in thyroid disorders. Thyroid 13: 755-760, 2003.

HAMPL R, SULCOVÁ J, BÍLEK R, HILL M: How short-term transdermal treatment of men with 7-oxodehydroepiandrosterone influence thyroid function. Physiol Res 55: 49-54, 2006.

LAPČÍK O, HAMPL R, HILL M, BIČÍKOVÁ M, STÁRKA L: Immunoassay of 7-hydroxysteroids: 1. radioimmunoassay of 7ß-hydroxydehydroepiandrosterone. J Steroid Biochem Mol Biol 67: 439-445, 1998.

LAPČÍK O, HAMPL R, HILL M, STÁRKA L: Immunoassay of 7-hydroxysteroids: 2. Radioimmunoassay of $7 \alpha-$ hydroxy-dehydroepiandrosterone. J Steroid Biochem Mol Biol 71: 231-237, 1999.

MORFIN R: Involvement of steroids and cytochromes $\mathrm{P}(450)$ species in the triggering of immune response. J Steroid Biochem Mol Biol 80: 273-290, 2002.

MULLER C, HENNEBERT O, MORFIN R: The native anti-glucocorticoid paradigm. J Steroid Biochem Mol Biol 100: 95-105, 2006.

PÉLISSIER MA, MULLER C, HILL M, MORFIN R: Protection against dextran sodium-sulfate induced colitis by dehydroepiandrosterone and 7alpha-hydroxy- dehydroepiandrosterone in the rat. Steroids 71: 240-248, 2005. 
ŠULCOVÁ J, HILL M, MAŠEK Z, ČEŠKA R, NOVÁČEK A, HAMPL R, STÁRKA L: Effects of transdermal application of 7-oxo-DHEA on the levels of steroid hormones, gonadotropins and lipids in healthy men. Physiol Res 50: 9-18, 2001.

VANDERPUMP MP, TUNBRIDGE WM: Epidemiology and prevention of clinical and subclinical hypothyroidism. Thyroid 12: 839-847, 2002.

WILSON GR, CURRY RW Jr: Subclinical thyroid disease. Am Fam Physician 72: 1517-1524, 2005. 